\title{
Análisis Morfométrico del Efecto de Estimulaciones de Láser Infrarrojo sobre Núcleos de Hepatocitos de Rata
}

\author{
Morphometric Analysis of the Effect of Infrared Laser Stimulation on Hepatocyte Nuclei in Rats
}

\author{
Ricardo Cornejo Uribe; Fernando Matamala Vargas; Héctor Silva Mella; \\ Orlando Garrido Oñate; Kurt Buchegger Mena \& Armin Jarpa Hidalgo
}

CORNEJO, U. R.; MATAMALA, V. F.; SILVA, M. H.; GARRIDO, O. O.; BUCHEGGER, M. K. \& JARPA, H. A. Análisis morfométrico del efecto de estimulaciones de láser infrarrojo sobre núcleos de hepatocitos de rata. Int. J. Morphol., 28(3):771-775, 2010 .

RESUMEN: De ratas Sprague Dawley tanto normales como irradiadas con dosis diarias de 1, 2, 4, 8, y $16 \mathrm{~J} / \mathrm{cm}^{2}$ durante 15 días emitidas por el láser AsGa equivalente a $904 \mathrm{~nm}$ previamente anestesiadas, fueron quirúrgicamente obtenidas muestras de hígado, las que posteriormente fueron procesadas para microscopía óptica, siendo estudiadas y sometidas a técnicas morfométricas utilizando aumentos de 1000X, con especial énfasis en cuantificar áreas de núcleos y nucleolos. El análisis de los resultados entre hepatocitos normales e irradiados revela que existen marcadas diferencias entre sus áreas tanto nucleares como nucleolares, concluyéndose que los efectos de estas dosis de radiación infrarroja provoca en los hepatocitos una drástica transformación en sus componentes y por ende en su funcionalidad, principalmente en la relativa a la síntesis proteica, representando el efecto de estas estimulaciones sobre este tipo celular de elevado metabolismo.

PALABRAS CLAVE: Láser infrarrojo; Hepatocitos; Morfometría.

\section{INTRODUCCIÓN}

El uso de las emisiones infrarrojas generadas por el diodo láser Arsenurio de Galio, se ha constituido en un método habitual en pro de obtener un sostenido alivio de una amplia gama de lesiones y afecciones articulares basado en su eficaz acción analgésica (Cetiner et al., 2006; Fikackova et al., 2007), antiinflamatoria (Fikackova et al., 2006) у cicatrizante (Kreisler et al., 2003; De Castro et al., 2001), mientras que a nivel celular induce una acentuada síntesis proteica (Simoes et al., 2009), de DNA (Pourzarandian et al., 2005), de ATP mitocondrial (Mochizuki-Oda et al., 2002; Oron et al., 2007) y de colágeno (Gomez-Villamandos et al., 1995; Cornejo et al., 2009a) como así mismo modulando la proliferación celular (Vinck et al., 2003; Mirzaei et al., 2007) las concentraciones de calcio citosólico (Mirodzhov et al., 1991; Smith et al., 2001) y la actividad de variadas enzimas (Cieslar et al., 1995; Kao \& Sheen 2003).

Sin embargo, en este contexto, el resultado de los efectos subcelulares de estas estimulaciones son escasas y poco descriptivas, además considerando que el estudio de la Biología Celular nos muestra que en medida que las células son estimuladas, sus componentes sufren modificaciones cualitativas, cuantitativas y topográficas, podría suponerse que mediante un análisis cuantitativo de placas histológicas y concordante con datos obtenidos de análisis morfométricos ultraestructurales revelara como los cambios morfológicos en estructuras nucleares se traducen en función celular, básicamente relacionada con síntesis proteica, en este modelo biológico (Junqueira \& Salles, 1975).

Por lo expuesto anteriormente, pareció importante precisar el rol ejercido por estimulaciones emitidas por el láser infrarrojo describiendo las respuestas celulares a nivel óptico y ultraestructural, relacionando estructuras nucleares con componentes asociados a síntesis proteica en hepatocitos de ratas irradiados con dosis infrarrojas crecientes por espacio de 15 días consecutivos, y verificar si las modificaciones que se efectúan conllevan a una alteración en su expresión génica. 


\section{MATERIAL Y MÉTODO}

A partir del hígado de ratas normales, irradiados mediante láser infrarrojo con dosis de 1, 2, 4, 8 y 16 Joules/ $\mathrm{cm}^{2}$ durante 15 días consecutivos, fueron obtenidas muestras las que se fijaron en formalina al $10 \%$ y posteriormente deshidratadas en batería creciente de alcoholes de 50 a $100 \%$, por 2 horas, para posteriormente, ser incluidas en Paraplast durante una noche. Se realizaron cortes seriados de $7 \mu \mathrm{m}$ de espesor, fijándose a portaobjetos albuminizados y dejando secar por 24 horas. El proceso de tinción requirió desparafinar con xilol por 40 minutos y proceder a la tinción nuclear mediante hematoxilina por 3 minutos y tinción citoplasmática con eosina por 5 minutos. Luego de un lavado con agua se deshidrataron los cortes con batería creciente de alcoholes otorgándoles transparencia mediante xilol fenicado por 5 minutos. Finalmente, se realizó el montaje de los cortes en medio Permount, dejando secar por 24 horas a temperatura ambiente.

Se obtuvieron micrografías ópticas de hepatocitos con aumento de hasta $1000 \mathrm{X}$ en un microscopio óptico asociado a una cámara de captura de imagen, modelo Moticam 2000, Motic 2.0 Megapixeles. Para el cálculo de áreas nucleares y nucleolares se utilizó el software Motic Images Plus ML, versión 2.0

\section{RESULTADOS}

Las estimulaciones con láser infrarrojo sobre hepatocitos de rata con dosis correspondiente a: 1, 2, 4, 8 y 16 Joules $/ \mathrm{cm}^{2}$ en comparación con hepatocitos normales, evidenciadas en la Figura 1, generan los siguientes datos morfométricos en relación a estructuras nucleares asociadas a síntesis proteica, analizadas en este estudio mediante microscopía óptica:

Se evidencia una directa relación entre dosis crecientes de láser infrarrojo asociado a una sostenida disminución en el área nuclear, y de igual manera se observa una relación directa entre el aumento de dosis infrarrojas con un evidente aumento de las áreas nucleolares, ambas observaciones logradas con una irradiación máxima de $8 \mathrm{~J} / \mathrm{cm}^{2}$. Situación que se muestra en la Tabla I.
Entre estimulaciones infrarrojas de 4 y $8 \mathrm{~J} / \mathrm{cm}^{2}$, se visualiza la mayor actividad celular de hepatocitos irradiados, datos que son refrendados con el aumento de las fracciones volumétricas obtenidas mediante microscopía electrónica de transmisión correspondiente a retículo endoplásmico rugoso, mitocondrias y disminución de los gránulos de glicógeno, además de un $90 \%$ correspondiente a eucromatina (Cornejo et al., 2009b) (Fig. 2).

En hepatocitos irradiados con dosis infrarrojas superiores a $8 \mathrm{~J} / \mathrm{cm}^{2}$, se observan notables modificaciones de las estructuras nucleares asociadas a síntesis proteica, manifestadas en un aumento del área nuclear y una disminución del área nucleolar, mientras que a nivel citosólico se visualiza disminución de las fracciones volumétricas de retículo endoplásmico rugoso y un aumento de las de glicógeno, todo lo cual se traduciría en una evidente inhibición de la actividad celular generada por estas estimulaciones infrarrojas elevadas.

\section{DISCUSIÓN}

Producto del análisis de los resultados se evidencia que el efecto de estimulaciones crecientes con láser infrarrojo sobre hepatocitos de rata, generan una progresiva disminución del área nuclear y de igual modo, un sostenido aumento de las áreas nucleolares, ambas situaciones determinarían cambios en la morfología, ultraestructura y funcionalidad, tanto a nivel nuclear como en la actividad citosólica.

Estas modificaciones anteriormente descritas se generarían, entre otros mecanismos, producto de una activa transcripción sintetizando diversos tipos de ácido ribonucleico (ARN). De igual manera, el constante aumento de las áreas nucleolares es debido, sin duda, a un incremento en la tasa de síntesis de ARN ribosomal. Estos hallazgos, producto de estimulaciones infrarrojas semejantes a las utilizadas son coincidentes a los descritos por Baibekov et al. (1992) visualizando incremento en los volúmenes nucleolares; Marques et al. (2004) donde los fibroblastos estudiados generan crecientes nucléolos; Koutna et al. (2003) comunicando activa síntesis de RNA.

En este mismo contexto, es decir, con estimulación correspondientes a $8 \mathrm{~J} / \mathrm{cm}^{2}$, se observa con claridad que las fracciones volumétricas de eucromatina corresponde a $90 \%$,

Tabla I. Áreas nucleares y nucleolares $\left(\mathrm{u}^{2}\right)$ correspondientes a hepatocitos de rata tanto normales como estimuladas con dosis crecientes de láser infrarrojo.

\begin{tabular}{ccccccc}
\hline & Normal & $1 \mathrm{~J} / \mathrm{cm}^{2}$ & $2 \mathrm{~J} / \mathrm{cm}^{2}$ & $4 \mathrm{~J} / \mathrm{cm}^{2}$ & $8 \mathrm{~J} / \mathrm{cm}^{2}$ & $16 \mathrm{~J} / \mathrm{cm}^{2}$ \\
\hline Área nuclear & 33 & 30 & 22 & 20 & 18 & 30 \\
\hline Área nucleolar & 3 & 10 & 11 & 13 & 10 & 9 \\
\hline
\end{tabular}



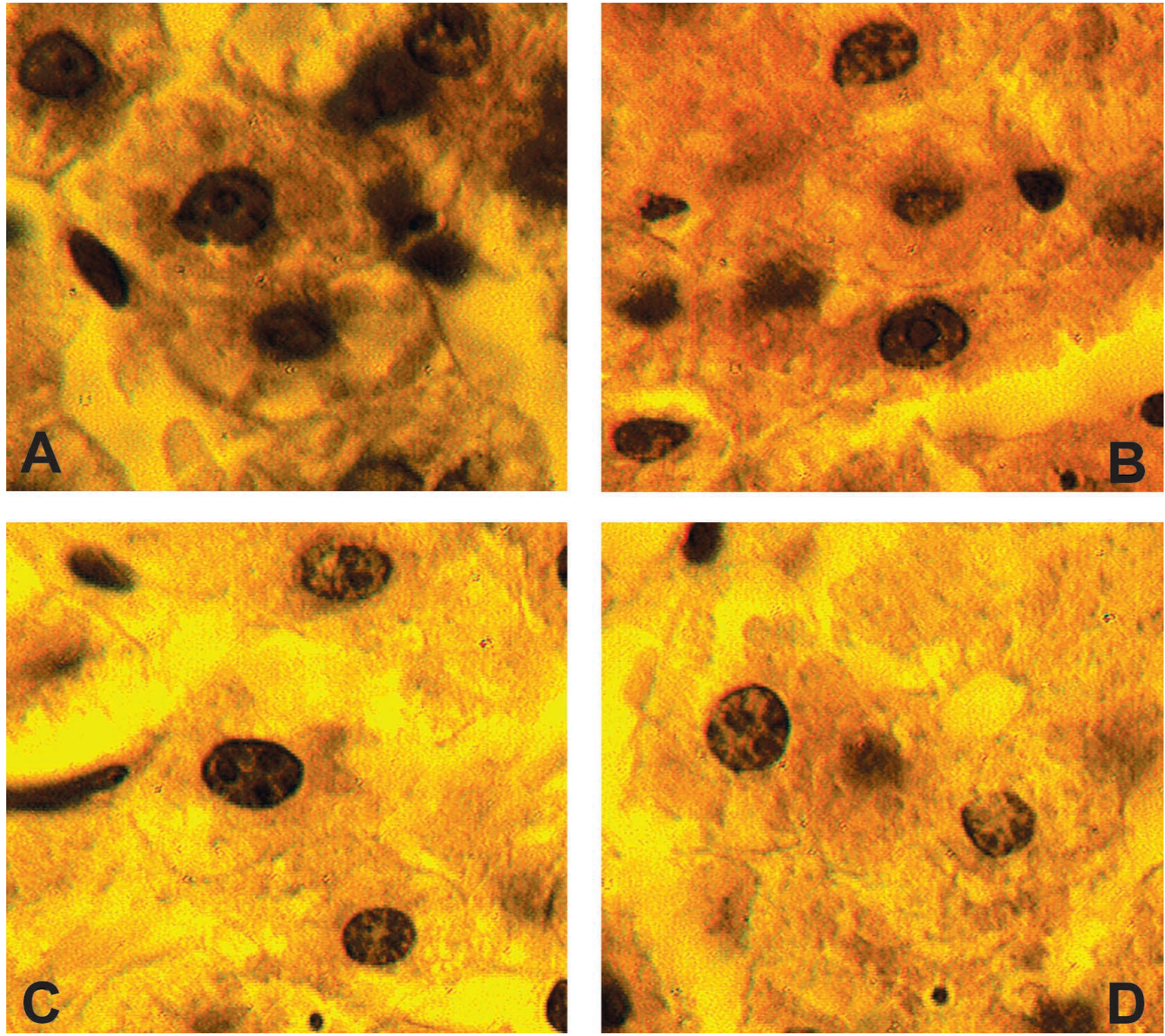

Fig. 1. Micrografias ópticas de hepatocitos de rata. A. Normales, B. Irradiados con dosis de $2 \mathrm{~J} / \mathrm{cm}^{2}$, C. Irradiados con $4 \mathrm{~J} / \mathrm{cm}^{2}$, D.irradiados con $8 \mathrm{~J} / \mathrm{cm}^{2} .1 .000 \mathrm{X}$.

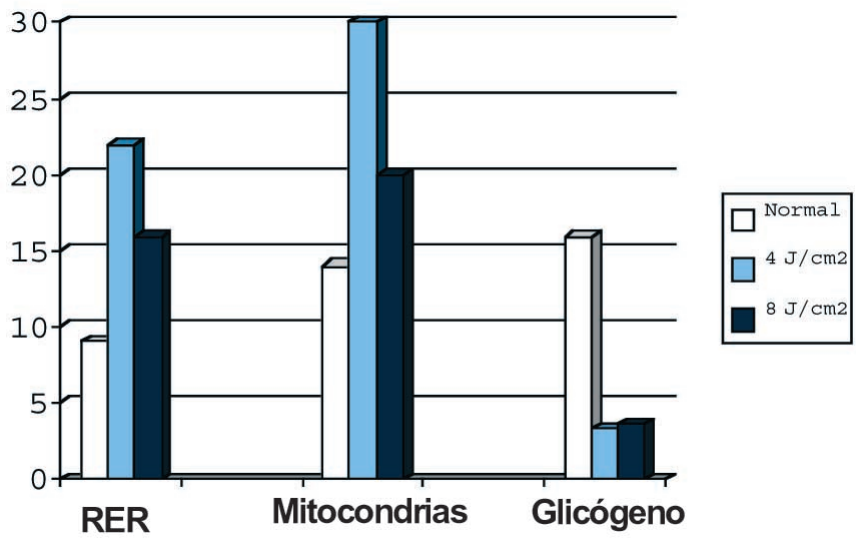

Fig. 2. Fracciones volumétricas $(\%)$ de R.E.R., Mitocondrias y licógeno evaluados en hepatocitos de rata normales e irradiados con láser infrarrojo de 4 y 8 Joules $/ \mathrm{cm}^{2}$. 
valor óptimo que indicaría que la expresión génica y la síntesis proteica es mayor que la efectuada tanto en el hepatocito normal, como en los estimulados con dosis menores (Cornejo et al., 2009b).

Por otro lado, es posible visualizar que el mayor desarrollo de componentes celulares, básicamente los involucrados en síntesis proteica: retículo endoplásmico rugoso (RER) y mitocondrias, es producto de las estimulaciones generadas con dosis entre 4 y $8 \mathrm{~J} / \mathrm{cm}^{2}$. Paralelamente es preciso considerar que los volúmenes de glicógeno disminuyen con dosis crecientes de láser indicando una constante degradación de glucosas citoplasmáticas para realizar procesos de glicólisis y posterior síntesis de ATP mitocondrial, obteniendo la energía necesaria para la correspondiente síntesis de proteínas Estos resultados son semejantes a los enunciados por Kao \& Sheen quienes demostraron que estas estimulaciones infrarrojas generan en hepatocitos de rata un notable incremento en la síntesis proteica, especialmente de glutatión.

Estos resultados aquí presentados definen que las dosis de emisiones infrarrojas entre 4 y $8 \mathrm{~J} / \mathrm{cm}^{2}$ son bené- ficas proclive a estimular el desarrollo de estructuras nucleares y citosólicas asociadas a síntesis proteica, y que, por el contrario, dosis mayores como $16 \mathrm{~J} / \mathrm{cm}^{2}$ generan disminución de constituyentes nucleares y citoplasmáticos, y por ende disminuyen la actividad celular, en este sistema biológico, hallazgos absolutamente coincidentes con los demostrados tanto por Hawkins \& Abrahamse (2007) como Houreld \& Abrahamse (2007) quienes demuestran que en fibroblastos estimulados con dosis entre 2.5 y $5 \mathrm{~J} /$ $\mathrm{cm}^{2}$ se produce un notable incremento tanto en la migración como en la proliferación de estas células y de igual modo, con dosis equivalente a $16 \mathrm{~J} / \mathrm{cm}^{2}$ se reduce la migración y proliferación llevando a un estado de stress celular, disminuyendo igualmente la síntesis de ATP y la viabilidad celular.

\section{AGRADECIMIENTOS}

Este trabajo es el resultado de las actividades de investigación realizadas en el proyecto DI-UFRO 08-0022, Universidad de La Frontera, Chile.

CORNEJO, U. R.; MATAMALA, V. F.; SILVA, M. H.; GARRIDO, O. O.; BUCHEGGER, M. K. \& JARPA, H. A. Morphometric analysis of the effect of infrared laser stimulation on hepatocyte nuclei in rats. Int. J. Morphol., 28(3):771-775, 2010.

SUMMARY: Liver samples were taken from previously anaesthetised Sprague Dawley rats, both normal and irradiated with daily doses of 1, 2, 4, 8, and $16 \mathrm{~J} / \mathrm{cm}^{2}$ applied over 15 days by AsGa laser equivalent to $904 \mathrm{~nm}$. These samples were then processed for optical microscopy. They were studied and subjected to morphometric techniques using 1000X magnification, placing special emphasis on the quantification of the areas of nuclei and nucleoli. An analytic comparison of the results between normal and irradiated hepatocytes reveals the existence of significant differences between both the nuclear and nucleolar areas studied, from which it is concluded that the effect of these doses of infrared radiation is to provoke a drastic transformation in the components of the hepatocytes, and therefore in their functioning, principally with respect to protein synthesis, and that this would be the effect of stimulation of this nature on this type of high-metabolism cell.

KEY WORDS: Infrared laser; Hepatocytes; Morphometry.

\section{REFERENCIAS BIBLIOGRÁFICAS}

Baibekov, I. M.; Vorozhei kin, V. M. \& Artykov, Sh. N. Effect of low-intensity infrared laser radiation on ultrastructure and proliferation of liver cells in experimental hepatitis and cirrhosis. Biull. Eksp. Biol. Med., 113(4):571-4, 1992.

Cetiner, S.; Sevil, A. \& Kahraman, R. Evaluation of lowlevel laser therapy in the treatment ot temporomandibular disorders. Photomed. Laser Surg., 24(5):637-41, 2006.

Cieslar, G.; Adamek, M.; Sieron, A. \& Kaminski, M. Influence of low-power laser radiation on the activity of some membraneous and mitochondrial enzymes of hepatocytes in rats. Proc. SPIE, 2323:546-50, 1995.

Cornejo, R.; Matamala, F.; Silva, H .\& Garrido, O. Morphometric and ultrastructural studies of effect infarred laser on rabbit temporo-mandibular joint fibroblasts. Braz. Arch. Biol. Technol., 52(3):611-6, 2009a.

Cornejo, R.; Matamala, F.; Silva, H .\& Garrido, O. Analisis morfométrico del efecto del láser infrarrojo sobre hepatocitos de rata. Int. J. Morphol. 27(3):831-6, 2009 b. 
De Castro, O.; Zucoloto, S.; Menegazzo, L. \& Granato, R. Laser enhancement in hepatic regeneration for partially hepatectomized rats. Lasers Surg. Med., 29(1):73-7, 2001.

Fikackova, H; Dostalova, T.; Navratil, L. \& Klaschka, J. Effectiveness of low-level laser therapy in temporomandibular joint disorders: a placebo-controlled study. Photomed Laser Surg., 25(4):297-303, 2007.

Fikackova, H.; Dostolava, T.; Vosicka, R. Peterova, V.; Navratil, L \& Lesak, J. Arthalgia of the temporomandibular joint and low-level laser therapy. Photomed Laser Surg 24(4):522-7, 2006.

Gomez-Villamandos, R. J.; Santisteban Valenzuela, J. M. \& Ruiz Calatrava, I.; Gomez-Villamandos, J. C. \& Avila Jurado, I. He-Ne laser therapy by fibroendoscopy in the mucosa of the equine upper airway. Laser Surg. Med. 16(2):184-8, 1995.

Hawkins, D. \& Abrahamse, H. Effect of multiple exposures of low-level laser therapy on the cellular responses of wounded human skin fibroblasts. Photomed. Laser Surg., 24(6):705-14, 2007.

Houreld, N. \& Abrahamse, H. In vitro exposure of wounded diabetic fibroblast cells to a helium-neon laser at 5 and 16 J/cm². Photomed. Laser Surg., 25(2):78-84, 2007.

Junqueira, L. C. U. \& Salles, L. Ultra-estrutura e funçao celular. Rio de Janeiro, Guanabara-Koogan, 1975. 44, 58.

Kao, M. \& Sheen, L. Effects of infrared and low-power laser irradiation on cell viability, glutathione and glutathionerelated enzyme activities in primary rat hepatocytes. $J$. Formos. Med. Assoc., 102(7):486-91, 2003.

Kreisler, M.; Christoffers A. B.; Willershausen, B. \& d'Hoedt, B. Effect of low-level GaAlAs laser irradiation on the proliferation rate of human periodontal ligament fibroblasts: An in vitro study. J. Clin. Periodontol., 30(4):353-8, 2003.

Koutná, M.; Janisch, R. \& Veselská, R. Effects of low-power laser irradiation on cell proliferation. Scripta Medica, 76(3):163-72, 2003.

Marques, M. M.; Pereira, A. N.; Fujihara, N. A.; Nogueira, F. N. \& Eduardo, C. P. Effect of low-power laser irradiation on protein synthesis and ultrastructure of human gingival fibroblasts. Lasers Surg. Med., 34:2605, 2004 .
Mirodzhov, G. K.; Barakaev, S. B.; Tkalicheva, L. I. \& Iakubov, I. I. Effect of low-energy laser irradiation on hepatocyte ploidy. Arkh. Patol., 53(11):54-6, 1991.

Mirzaei, M.; Bayat, M.; Mosafa, N.; Mohsenifar, Z.; Piryaei, A.; Farokhi, B.; Rezaei, F.; Sadeghi, Y. \& Rakhshan, M. Effect of low-level laser therapy on skin fibroblasts of streptozotocin-diabetic rats. Photomed Laser Surg., 25(6):519-25, 2007.

Mochizuki-Oda, N.; Kataoka, Y.; Cui, Y.; Yamada, H.; Heya, M., \& Awazu, K. Effects of near-infra-red laser irradiation on adenosine triphosphate and adenosine diphosphate contents of rat brain tissue. Neurosci. Lett., 323:207-9, 2002.

Oron, U.; Ilic, S.; De Taboada, L. \& Streeter, J. Ga-As (808 $\mathrm{nm}$ ) laser irradiation enhances ATP production in human neuronal cells in culture. Photomed. Laser Surg., 25(3):180-2, 2007.

Puorzarandian, A.; Watanabe, H.; Ruwanpura, S. M.; Aoki, A. \& Ishikawa, I. Effect of low-level Er:YAG laser irradiation on cultured human gingival fibroblasts. $J$. Periodontol., 76(2):187-93, 2005.

Simoes, A.; Siqueira, W. L.; Lamers, M. L.; Santos, M. F.; Eduardo C. de P. \& Nicolau, J. Laser phototherapy effect on protein metabolism parameters of rat salivary glands. Lasers Med. Sci., 24:202-8, 2009.

Smith, N. I.; Fujita, K.; Kaneco, T.; Kato, O.; Nakamura, O.; Takamatsu, T. \& Kawata, S. Generation of calcium waves in living cells by pulsed-laser-induced photodisruption. Appl. Phys. Lett., 79(8):1208-10, 2001.

Vinck, E. M.; Cagnie, B. J.; Cornelissen, M. J.; Declercq, H. A. \& Cambier, D. C. Increased fibroblast proliferation induced by light emitting diode and low power laser irradiation. Laser Med. Sci., 18(2):95-9, 2003.

\section{Dirección para correspondencia: \\ Prof. Dr. Ricardo Cornejo Uribe \\ Facultad de Medicina \\ Universidad de La Frontera \\ Casilla 54-D, Temuco \\ CHILE}

Email: rcornejo@ufro.cl

Recibido : 22-04-2010

Aceptado: 17-06-2010 
